\title{
Sympathetic Fiber Sprouting in Inflamed Joints and Adjacent Skin Contributes to Pain-Related Behavior in Arthritis
}

\author{
Geraldine Longo, ${ }^{1 *}$ Maria Osikowicz, ${ }^{1 *}$ and Alfredo Ribeiro-da-Silva ${ }^{1,2}$ \\ ${ }^{1}$ Department of Pharmacology and Therapeutics, McGill University, Montreal, Quebec H3G 1Y6, Canada, and 2Department of Anatomy and Cell Biology, \\ McGill University, Montreal, Quebec H3A 2B2, Canada
}

\begin{abstract}
Although chronic pain is the most common symptom of arthritis, relatively little is known about the mechanisms driving it. Recently, a sprouting of autonomic sympathetic fibers into the upper dermis of the skin, an area that is normally devoid of them, was found in the skin following chronic inflammation of the rat hindpaw. While this sprouting only occurred when signs of joint and bone damage were present, it remained to be clarified whether it was a consequence of the chronic inflammation of the skin or of the arthritis and whether it also occurred in the joint. In the present study, we used a model of arthritis in which complete Freund's adjuvant (CFA) was injected into the rat ankle joint. At 4 weeks following CFA treatment, there was an increase in sympathetic and peptidergic fiber density in the ankle joint synovium. We also observed a sympathetic, but not peptidergic, fiber sprouting in the skin over the joint, which may be a consequence of the increased levels of mature nerve growth factor levels in skin, as revealed by Western blot analysis. The pharmacological suppression of sympathetic fiber function with systemic guanethidine significantly decreased the pain-related behavior associated with arthritis. Guanethidine completely suppressed the heat hyperalgesia and attenuated mechanical and cold hypersensitivity. These results suggest that transmitters released from the sprouted sympathetic fibers in the synovial membrane and upper dermis contribute to the pain-related behavior associated with arthritis. Blocking the sympathetic fiber sprouting may provide a novel therapeutic approach to alleviate pain in arthritis.
\end{abstract}

\section{Introduction}

Arthritis is a debilitating disease affecting $21 \%$ of the U.S. population (Lawrence et al., 2008). Patients suffering from osteoarthritis and rheumatoid arthritis make up $42 \%$ of all individuals afflicted with chronic pain (Breivik et al., 2006). The main symptom underlying arthritis is pain due to bone degeneration and inflammation affecting the joints. Although many symptomatic treatments do exist, there is no effective drug therapy to prevent or revert the underlying pathology.

Nociceptive fibers are responsible for relaying pain-related peripheral information from mechanical, thermal, and chemical stimuli to the CNS. The glabrous skin of the rat hindpaw is innervated by sensory and postganglionic sympathetic fibers. The

Received Dec. 17, 2012; revised May 9, 2013; accepted May 10, 2013.

Author contributions: G.L., M.O., and A.R.-d.-S. designed research;G.L., M.O., and A.R.-d.-S. performed research; G.L. and M.O. analyzed data; G.L., M.O., and A.R.-d.-S. wrote the paper.

This research was funded by grants from the Canadian Institutes of Health Research (CIHR) (MOP 79411), the Louise and Alan Edwards Foundation, and MITACS Accelerate Quebec. The authors thank Manon St. Louis for her laboratory expertise; Magalie Millecamps for teaching us the intra-articular injection technique; Gary J. Bennett for allowing us to use his animal behavioral testing facility; and Dr. PatrickW. Mantyh and Magdalena Kaczmarska, from the University of Arizona, for assistance with the processing of hard tissues for immunohistochemistry.

*G.L. and M.0. contributed equally to this research.

The authors declare no competing financial interests.

Correspondence should be addressed to Dr. Alfredo Ribeiro-da-Silva, Department of Pharmacology and Therapeutics, McGill University, 3655 Promenade Sir-William-0sler, Montreal, QC H3G 1Y6, Canada. E-mail: alfredo.ribeirodasilva@mcgill.ca.

DOI:10.1523/JNEUROSCI.5784-12.2013

Copyright $\odot 2013$ the authors $\quad 0270-6474 / 13 / 3310066-09 \$ 15.00 / 0$ peptidergic nociceptive fibers, recognized by means of immunoreactivity for the neuropeptides substance $\mathrm{P}$ and calcitonin generelated peptide (CGRP), innervate mostly the dermis and to a minor extent the epidermis. (Yen et al., 2006; Taylor et al., 2009). Joints and the periosteum are also innervated by nociceptive primary afferents and sympathetic fibers (Hara-Irie et al., 1996; Mach et al., 2002).

Previous research has shown that a subcutaneous injection of complete Freund's adjuvant (CFA) into the rat hindpaw is a model of polyarthritis suitable to study chronic pain and neuronal plasticity (Nagakura et al., 2003). In this model, there was persistent inflammation of soft tissues and joints; signs of joint damage developed at 2 weeks post-CFA (Vermeirsch et al., 2007; Almarestani et al., 2011). Interestingly, our group observed an ectopic presence of sympathetic fibers in the upper dermis of the skin (Almarestani et al., 2008). In normal conditions, postganglionic sympathetic fibers innervate blood vessels in the lower dermis (Yen et al., 2006); however the ectopic sympathetic fibers were not associated with the vasculature. Instead, they wrapped around peptidergic fibers (Almarestani et al., 2008). It was not known whether these changes in the innervation were a consequence of the chronic skin inflammation or resulted from the arthritis itself. This novel arrangement may favor sympathetic/ sensory fiber interactions and may contribute to the hypersensitivity observed in arthritis.

The ectopic sprouting of sympathetic fibers is likely trophic factor mediated. One neurotrophic factor proposed to play a 
major role in this sprouting is nerve growth factor (NGF). During early postnatal development, both sympathetic and primary afferent peptidergic fibers are dependent on NGF for neurotrophic support, whereas in adulthood NGF is required for survival of sympathetic neurons and proper phenotype maintenance in peptidergic afferents (Levi-Montalcini, 1987; Lewin and Barde, 1996). NGF plays an important role in mediating the inflammatory responses after tissue injury (McMahon et al., 2006). Here, we investigated the changes in innervation of the synovial membrane and skin over the ankle joint in an inflammatory arthritis model and correlated these changes with pain-related behavior. We investigated a possible role of the sprouted sympathetic fibers in pain by blocking their function pharmacologically with guanethidine. Finally, we studied the changes in protein levels of both the precursor and mature forms of NGF in this animal model.

\section{Materials and Methods}

Animals. Experiments were performed on male Sprague Dawley rats (Charles River) weighing 275-300 g at the beginning of the study. The entire experimental design was performed following the guidelines contained in the Care and Use of Experimental Animals of the Canadian Council on Animal Care. Moreover, all studies were approved by the Faculty of Animal Care Committee of McGill University and were conducted in accordance with the Guidelines for Animal Research by the International Association for the Study of Pain (Zimmermann, 1983). All animals were kept on a $12 \mathrm{~h}$ light/dark cycle; food and water were available ad libitum. The animals were housed in cages with soft bedding. Animals were divided into three separate cohorts for morphological analyses, biochemical studies, and pharmacological studies.

Model of mono-arthritis. Arthritis was induced by means of CFA. Animals were anesthetized with $5 \%$ isoflurane in $\mathrm{O}_{2}$ and injected with $40 \mu \mathrm{l}$ of CFA, containing $150 \mu \mathrm{g}$ of Mycobacterium butyricum, intra-articularly into the tibial-tarsal joint of the right-hand paw (Butler et al., 1992). Control (sham) animals underwent the same procedure but were injected with the same volume of vehicle (mineral oil).

Tissue edema of the ankle joint. One group of rats was used to assess the extent of tissue edema surrounding the inflamed joint in this model. Ankle joints were dissected out, weighed, and dried in an oven at $60^{\circ} \mathrm{C}$ for $24 \mathrm{~h}$. Edema in the ankle joint was determined as the loss in weight of each ankle joint after this dehydration procedure.

Behavioral tests. Pain-related behavior was assessed using the von Frey, Hargreaves, and acetone tests. Before any behavioral testing, animals were habituated to the testing environment. The baseline reaction values were measured $1 \mathrm{~d}$ before vehicle or CFA injection and were as follows: $21.6 \pm 1.6 \mathrm{~g}$ for the von Frey test; $12.4 \pm 1.1 \mathrm{~s}$ for the Hargreaves test; and 0 for the acetone test.

Mechanical allodynia (von Frey test). Mechanical allodynia in rats was measured using a series of calibrated von Frey filaments (Stoelting), ranging from 0.6 to $26 \mathrm{~g}$. Animals were placed in plastic cages with a wire-mesh floor. The von Frey filaments were applied in ascending order to the midplantar surface of the ipsilateral hindpaw through the mesh floor. Each probe was applied to the foot until it bent. The time interval between consecutive filament administrations was at least $5 \mathrm{~s}$. The calculations were performed as described previously (Osikowicz et al., 2008).

Cold allodynia (acetone test). Cold allodynia was assessed using the acetone drop method (Choi et al., 1994). In the test environment described above, a $50 \mu \mathrm{l}$ droplet of acetone was applied to the midplantar region of the hindpaw using a micropipette. Responses within the first 20 s were scored as follows: 0 , no response; 1 , one rapid hindpaw flick/ stamp; 2, two or more hindpaw flicks/stamps; 3, periods of flicking/ stamping with licking of plantar hindpaw (Flatters and Bennett, 2004). Acetone application was repeated three times for each hindpaw, with a 3 min interval between each application. For each rat, the sum of the three scores was then used for data analysis.

Thermal hyperalgesia (Hargreaves test). The pain threshold to high temperature was tested using the plantar test (Hargreaves Apparatus,
Type 7370; Ugo Basile). Rats were placed into individual plastic cages with glass floors $5 \mathrm{~min}$ before the experiment. A noxious thermal stimulus was focused through the glass onto the plantar surface of a hindpaw until the animal lifted the paw away from the heat source. The paw withdrawal latency was automatically displayed to the nearest $0.1 \mathrm{~s}$. A cutoff latency of $20 \mathrm{~s}$ was used to avoid tissue damage. The latency of nociceptive reaction was measured in seconds under baseline conditions and weekly after CFA or vehicle injection.

Western blot. Four weeks after CFA or vehicle injection, the rats were decapitated, and the glabrous skin from the hindpaws was collected, frozen in the liquid nitrogen, and stored in the freezer $\left(-80^{\circ} \mathrm{C}\right)$ for further processing. Tissue samples were homogenized in RIPA buffer $(1 \%$ NP-40, $1 \%$ sodium deoxycholate, $0.1 \%$ SDS, $150 \mathrm{~mm} \mathrm{NaCl}, 25 \mathrm{ml}$ Tris$\mathrm{HCl}, \mathrm{pH}$ 7.6) containing protease inhibitors (Complete; Roche Molecular Biochemicals) and cleared by centrifugation (13,000 rpm for $50 \mathrm{~min}$ at $\left.4^{\circ} \mathrm{C}\right)$. Protein concentration of the supernatant was determined using the BCA Protein Assay Kit (Sigma). Homogenates ( $20 \mu \mathrm{l}$ of $50 \mu \mathrm{g}$ of total protein) were resolved in $4-12 \%$ polyacrylamide gels and transferred into a nitrocellulose membrane (Bio-Rad). The blots were blocked in Tris-buffered saline-Tween 20 (TBS-T) containing 5\% nonfat dry milk at room temperature for $1 \mathrm{~h}$ on a rotomixer. Nitrocellulose membranes were probed with primary antibodies specific for NGF (1:500; Santa Cruz Biotechnology). Blots were incubated overnight at $4^{\circ} \mathrm{C}$ with the primary antibody. After primary antibody incubation, membranes were washed $3 \times 10 \mathrm{~min}$ in TBS-T. This was followed by incubation for $2 \mathrm{~h}$ at room temperature with a peroxidase-conjugated goat anti-rabbit Ig antibody (1:2500) (Jackson ImmunoResearch). The membranes were washed $3 \times$ $10 \mathrm{~min}$ in TBS-T. The immunoreactive (IR) bands were visualized with an ECL kit (PerkinElmer) and using Kodak Biomax XAR imaging film. The immunoreactive bands were quantified by densitometry of the films using an MCID M4 image analysis system (Imaging Research). Membranes were rinsed and reprobed with a mouse anti- $\beta$-actin antibody (1:40,000; Sigma) diluted in 5\% milk in TBS-T for $1 \mathrm{~h}$ at room temperature, washed with TBS-T, and incubated with a peroxidase-conjugated donkey anti-mouse IgG (1:5000; Santa Cruz Biotechnology) in 5\% dry milk in TBS-T for $1 \mathrm{~h}$. The membranes were washed, and the signal was detected and qualified as described above. The protein levels of the precursor (proNGF) and mature ( $\mathrm{mNGF)} \mathrm{forms} \mathrm{of} \mathrm{NGF} \mathrm{were} \mathrm{normalized} \mathrm{to}$ the $\beta$-actin levels for each sample.

Immunohistochemistry. Animals were perfused with histological fixatives at 1,2,3, or 4 weeks after CFA or oil injections. For this, they were deeply anesthetized with Equithesin $(6.5 \mathrm{mg}$ of chloral hydrate and $3 \mathrm{mg}$ of sodium pentobarbital in a volume of $0.3 \mathrm{ml}$, i.p., per $100 \mathrm{~g}$ of body weight) and then perfused through the left cardiac ventricle with $100 \mathrm{ml}$ of perfusion buffer, followed by $500 \mathrm{ml}$ of $4 \%$ paraformaldehyde in $0.1 \mathrm{M}$ phosphate buffer (PB), pH 7.4, at room temperature for $30 \mathrm{~min}$. Subsequently, the glabrous skin and ankle joints were extracted and post-fixed in the same fixative for $1 \mathrm{~h}$ at $4^{\circ} \mathrm{C}$. A benefit of using this model of arthritis compared with the knee joint model is that we can use paw withdrawal thresholds to test for pain-related behavior induced by the arthritis. Ankle joints underwent a decalcification step in 10\% EDTA in $\mathrm{dH}_{2} \mathrm{O}$ for 3 weeks. Subsequently, tissue was cryoprotected in $30 \%$ sucrose in $\mathrm{PB}$ overnight at $4^{\circ} \mathrm{C}$ for later immunohistochemical processing.

To study the glabrous skin, $50-\mu \mathrm{m}$-thick sections were cut using a cryostat. All sections were collected as free-floating in PBS with $0.2 \%$ Triton X-100 (PBS-T). Ankle joint sections were cut at $16 \mu \mathrm{m}$ in thickness and attached directly onto gelatin-subbed slides for immunostaining. The tissue sections were incubated for $1 \mathrm{~h}$ at room temperature in $10 \%$ normal goat serum (Invitrogen) in PBS to block unspecific labeling. To detect immunoreactivity of the peptidergic and sympathetic fiber populations, the sections were then incubated at $4^{\circ} \mathrm{C}$ for $24 \mathrm{~h}$ using a rabbit anti-CGRP (Sigma-Aldrich) antibody or rabbit anti-vesicular monoamine transporter-2 (VMAT-2) antibody (Synaptic Systems) at dilutions of 1:2000 and 1:7500, respectively. After several rinses in PBS-T, the sections were incubated for $2 \mathrm{~h}$ at room temperature with secondary antibodies, a goat anti-rabbit IgG conjugated to either Alexa Fluor 488 or Alexa Fluor 594 (Invitrogen) at a dilution of 1:400 in PBS-T. For double labeling of CGRP and VMAT-2, sections were processed as described above except that we used a mixture of a guinea pig anti-CGRP antibody 
at a 1:8000 dilution (Peninsula) and of the VMAT-2 antibody; after washing, we used a mixture of an anti-guinea pig IgG antibody conjugated to Alexa Fluor 488 (1:800; Invitrogen) with the anti-rabbit IgG conjugated to Alexa Fluor 594. Last, the sections were washed, mounted on gelatin-subbed slides, air-dried, and coverslipped with an anti-fading mounting medium (Aqua PolyMount; Polysciences). Some sections were processed omitting the primary antibody; no specific staining was observed. Slides were stored at $4^{\circ} \mathrm{C}$ until examined. Before quantitative analyses, all the slides were coded so that the person who performed the quantification was completely blinded regarding the experimental groups. Codes were broken only after the quantification was completed.

CGRP-immunoreactive fiber quantification. We studied the innervation of the upper dermis of the glabrous skin of the hindpaw and the ankle joint synovial membrane on the ipsilateral side of the injection. As in previous studies from our laboratory, we considered the upper dermis as a band $150 \mu \mathrm{m}$ in thickness residing immediately below the dermalepidermal junction (Yen et al., 2006). All the material used for quantification was single labeled. For the measurement of the CGRP-IR fiber innervation density, digital images were captured with a high-resolution camera attached to a Zeiss Axioplan 2e imaging fluorescence microscope, using a PlanFluotar $40 \times$ objective. This microscope is connected to a computer equipped with the Zeiss Axiovision 4.8 software. For each rat, three sections of the skin and three sections of the ankle joint were selected at random, and in each section six non-overlapping micrographs of the upper dermis and/or synovial membrane were taken, for a total of 18 images per region per animal. Images were exported in TIFF format for analysis using an MCID Elite image analysis system (Imaging Research). The region corresponding to the upper dermis was outlined with the tracing tool of the software. CGRP-IR fibers were detected and converted to 1 pixel in thickness to measure the total fiber length (in micrometers) per scan area (in square micrometers). For statistical comparisons, we used a one-way ANOVA with Dunnett's post hoc test. Statistical significance was set at $p<0.05$.

Sympathetic fiber quantification. VMAT-2-IR fibers in the ankle joint synovial membrane were quantified using the same approach as for the CGRP-IR fibers (described above). The quantification of the changes in autonomic innervation of the glabrous skin was performed using a different approach from that used for sensory fibers. Since the density of sympathetic fibers in the upper dermis is low, it is very difficult to measure directly. Therefore, we counted on each of six sections per animal all VMAT-2-IR fibers within the upper dermis. The mean number of fibers in the upper dermis per square micrometer was compared between groups using a one-way ANOVA and a Dunnett's post hoc test, with a statistical significance set at $p<0.05$.

Drug administration for sympathetic block. For the sympathetic block study, vehicle (saline) or $30 \mathrm{mg} / \mathrm{kg}$ guanethidine sulfate (Santa Cruz Biotechnology) were administered intraperitoneally twice with a $24 \mathrm{~h}$ interval, at the 2 and 4 weeks post-CFA time points (Xanthos et al., 2008). All three behavioral tests described above were performed at baseline (day 0), 2 or 4 weeks postinjection (predrug in sham and CFA), and $4 \mathrm{~h}$ after administration of the second dose of guanethidine on either day 15 or 30 (postdrug). Animals were divided into the following different groups: sham + vehicle, sham + guanethidine, CFA + vehicle, and CFA + guanethidine. Several studies show evidence that a single dose of systemic guanethidine is sufficient for long-term sympathetic blockade (Maxwell et al., 1960; Kim et al., 1993). Data from the animals in the predrug group $(\mathrm{CFA}+$ vehicle and CFA + guanethidine) were pooled together since no statistically significant difference was detected.

Statistical analyses. All statistical tests were performed using GraphPad Prism version 5 for Windows (GraphPad Software). All values are expressed as mean \pm SEM. To compare for changes in the innervation of the skin, groups were compared by one-way ANOVA with Dunnett's post hoc analysis. To analyze changes in the innervation of the synovial membrane, an unpaired $t$ test was performed to compare the control group to the CFA group. Group comparisons for the guanethidine study were analyzed using a one-way ANOVA with Bonferroni's multiple-comparison test to compare the CFA groups (vehicle vs guanethidine).

\section{Results}

\section{Quantification of edema around the ankle joint}

To measure ankle edema, ankle joints were weighed before and after dehydration in an oven at $60^{\circ} \mathrm{C}$ for $24 \mathrm{~h}$. Values represent the change in weight of the joint (plus surrounding tissues). The mean weight loss in sham animals was $0.94 \pm 0.07 \mathrm{~g}$, whereas in CFA-injected rats it was $2.81 \pm 0.4 \mathrm{~g}$ at the 4 week time point $(p<$ $0.005)$. These values revealed that the CFA-treated rats had significantly more fluid in the tissues surrounding the ankle joints than the sham rats. In contrast, the values from the contraleral side in both sham and CFA-treated rats were not different from the ipsilateral side in sham animals.

\section{Development of allodynia and hyperalgesia in rats following CFA administration}

In the behavioral tests, all CFA-treated rats exhibited strong mechanical and cold allodynia as well as thermal hyperalgesia as measured on the ipsilateral paw using the von Frey, acetone, and Hargreaves tests, respectively, at 1, 2, 3, and 4 weeks post-CFA (Fig. $1 A-C$ ). Behavioral tests were also performed on the contralateral hindpaw; however, no significant differences in sensitivity to mechanical and thermal stimulation were observed between experimental and control animals. The thresholds in the sham group were not changed during the observation period (Fig. 1), except with von Frey hairs at 1 week, when a mild hypersensitivity was observed (Fig. 1A).

\section{Changes in the pattern of innervation of the synovial membrane}

The innervation of the synovial membrane ipsilateral to the injection of CFA or oil (sham) was studied at the 4 week time point (Fig. 2). In control rats, there was a sparse innervation by sympathetic (VMAT-2-IR) fibers (Fig. 2A) and CGRP-IR fibers (Fig. $2 B)$. In CFA-injected animals, there was a significant increase in the density of both VMAT-2-IR fibers $\left({ }^{* *} p<0.005\right)$ (Fig. $2 D$ ) and CGRP-IR fibers $\left.{ }^{* *} p<0.005\right)$ (Fig. $2 E$ ). In CFA-injected rats, the VMAT-2-IR fibers of the synovium were in close proximity to CGRP-IR fibers, wrapping around each other (Fig. $2 F$, white arrows).

\section{Changes in the pattern of innervation of the thick skin over the ankle joint}

The CGRP-IR (peptidergic) fiber innervation density remained unchanged at all post-CFA injection time points studied (Fig. 3). On the other hand, there was an ectopic presence of sympathetic fibers (as detected by VMAT-2 immunoreactivity) in the upper dermis beginning at 2 weeks post-CFA injection (Fig. 4). The number of VMAT-2-IR fibers per unit area in the upper dermis was threefold higher in CFA-injected rats, compared with sham animals, at the 4 week time point $\left({ }^{* *} p<0.05\right)$. Similarly to what was observed in the synovium, the VMAT-2-IR fibers in the upper dermis were in close proximity to CGRP-IR fibers, and some appositions of the two fiber populations were seen (Fig. 5, white arrows).

\section{Changes in NGF protein levels in the glabrous skin of rats 4 weeks following CFA administration}

Western blot analyses of the glabrous skin samples were performed at 4 weeks after CFA or oil injections. Both proNGF and mNGF were identified based on their molecular weights; proNGF migrated close to $40 \mathrm{kDa}$, and mNGF migrated at $14 \mathrm{kDa}$ (Fig. 6). The localization of these bands is consistent with what is 

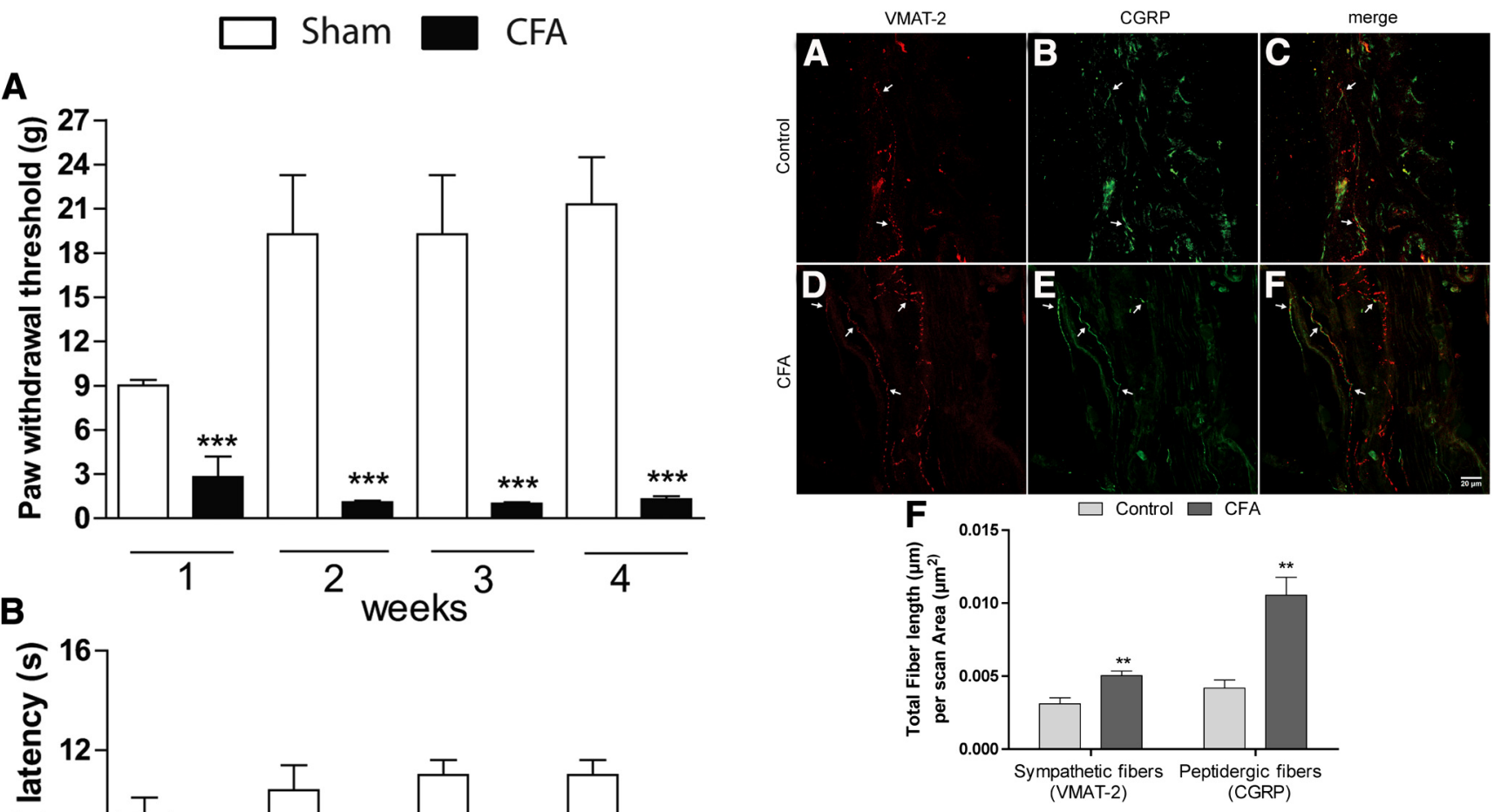

Figure 2. $A-F$, Changes in the nerve fiber innervation pattern of the synovial membrane of the ipsilateral ankle joint at 4 weeks after vehicle $(\boldsymbol{A}-\boldsymbol{C})$ or CFA (D-F) injection. $\mathbf{G}$, CFA-injected animals displayed a net increase in both VMAT-2-IR (red) and CGRP-IR (green) fiber density when compared with the control group, as can be observed in the confocal images and in the graph. In $\boldsymbol{C}$ and $\boldsymbol{F}$, note that VMAT-2-IR and CGRP-IR fibers in control and CFA-injected animals sometimes are in close apposition to each other (white arrows) and that such appositions are more abundant in the CFA group $(\boldsymbol{F})$. Images represent confocal microscope Z-stacks of optical sections. Scale bar, $20 \mu \mathrm{m}$. See Materials and Methods for details of quantification. Comparisons between CFA and control groups were done by means of an unpaired $t$ test. Values represent means \pm SEM.

C

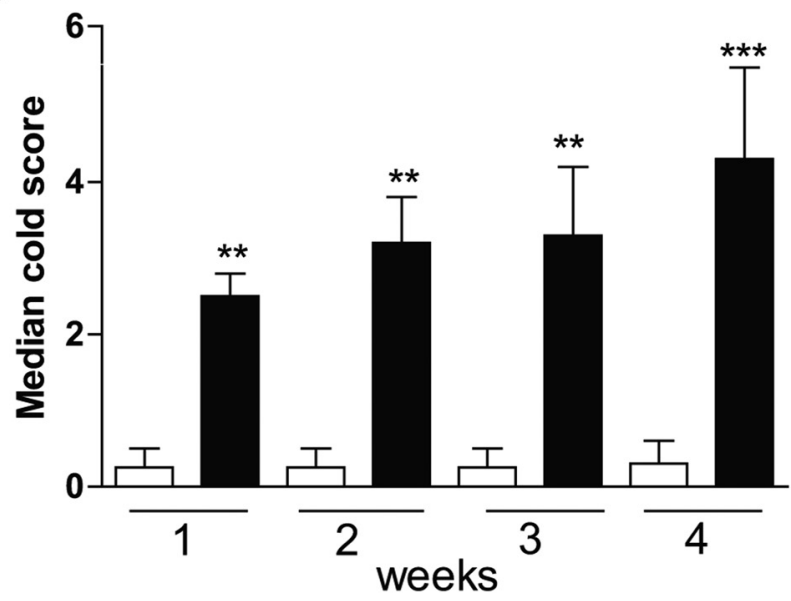

Figure 1. Pain-related behavioral analysis of sham and CFA-treated animals. A-C, In the behavioral tests, all CFA-treated rats exhibited strong mechanical and cold allodynia as well as thermal hyperalgesia as measured on the ipsilateral paw in the von Frey, acetone, and Hargreaves tests, respectively, atall experimental time points (1,2,3, and 4weeks). The pain thresholds in the sham group were not changed from baseline at any time point, except when applying von Frey hairs at the 1 week time point, where a significant lowering of the threshold was observed, possibly as a result of the solvent injection in the joint $(A)$. Statistical analysis was performed using one-way ANOVA and Bonferroni's multiple-comparison test. Error bars represent the SEM.

described in other publications using the same antibodies (Bruno and Cuello, 2006; Allard et al., 2012) and is aligned with the corresponding bands from positive controls (for NGF, mouse submandibular gland extracts; data not shown). Our data re- vealed an increase in the protein levels of $\mathrm{mNGF}$ in the ipsilateral paw compared with the contralateral paw $(0.49 \pm 0.02$ vs $0.4 \pm$ 0.01 ; Fig. $6 \mathrm{~A}$ ) and also when compared with ipsilateral paw samples from oil-treated rats $(0.49 \pm 0.02$ vs $0.39 \pm 0.01)$. However, no significant changes in the protein levels of proNGF were detected by Western blot analysis in the ipsilateral paw when compared with the results from the contralateral paw $(0.74 \pm 0.03$ vs $0.76 \pm 0.13$; Fig. $6 B$ ) and from the ipsilateral paw of oil-treated rats $(0.74 \pm 0.03$ vs $0.75 \pm 0.03)$.

\section{Effect of sympathetic block on pain behavior following CFA administration}

Figure 7 illustrates the pain-related behavioral data from rats before and after guanethidine or vehicle treatment at 2 and 4 weeks post-CFA or oil injection. Guanethidine did not have an effect on the behavioral pattern of sham (oil-injected) animals (data not shown). In addition, no statistical difference was found when comparing the sham groups (baseline, and before/after vehicle and guanethidine treatment) to the baseline threshold levels of the CFA groups. Statistical assessment was performed using a one-way ANOVA with Bonferroni's post hoc test comparing baseline values against all other treatments. At 2 weeks following CFA injection, guanethidine injections did not have any significant effect on pain-related behavior (Fig. $7 A-C$ ). Interestingly, at the 4 week time point, the CFA + guanethidine group demonstrated a significant difference compared with the CFA + vehicle group in all three behavioral parameters tested. Indeed, mechan- 
ical (Fig. 7A) and cold allodynia (Fig. 7B) thresholds partially returned to baseline post-guanethidine treatment ( $\sim 50 \%$ reversal). Importantly, the CFA + guanethidine group showed no significant difference compared with baseline levels in the Hargreaves test, indicating that the heat hyperalgesia was completely reversed (Fig. 7C). There was a significant difference when comparing both CFA groups (vehicle vs guanethidine) in all three behavioral tests, indicating that blocking sympathetic function increased the painrelated thresholds. These results correlate with the changes observed in the sympathetic fiber population in the glabrous skin surrounding the ankle joint at 4 weeks post-CFA injection.

\section{Discussion}

We used a well established mono-arthritis model consisting of the unilateral intraarticular injection of CFA in the ankle joint. We show that the CFA-injected animals displayed mechanical and cold allodynia as well as heat hyperalgesia, which was still detected at 4 weeks postinjection. Furthermore, we detected a sprouting of sympathetic fibers both in the ankle joint synovium and in adjacent skin. The density of nociceptive peptidergic nerve fibers was elevated in the synovial membrane but not in skin. The suppression of sympathetic fiber function with guanethidine partially alleviated the pain-related behavior. Protein levels of mNGF, but not proNGF, were elevated in CFA-treated rats.

Although we observed similar ipsilateral pain-related hypersensitivity at 1,2, 3, and 4 weeks post-CFA, mechanisms likely differ with the time points. Indeed, inflammatory mechanisms prevail at 1 week post-CFA. At this stage, inflammatory mediators, such as cytokines IL- $1 \beta$ and TNF- $\alpha$, released into the synovium and surrounding tissues, maintain nociceptor hyperexcitability (Miller et al., 2009). In the polyarthritis CFA model, joint and bone damage were present as from 2 weeks post-CFA (Henry, 2004; Almarestani et al., 2011). Therefore, at later time points there is a component of the hyperexcitability associated with the destruction of cartilage and bone. Additionally, we observed plastic changes in the innervation pattern of the ankle joint synovium and glabrous skin surrounding the joint. At 4 weeks post-CFA, we found a significantly denser innervation of the synovial membrane by sympathetic fibers. This nerve fiber remodeling can be explained by the surge of proinflammatory mediators and growth factors attributed to synovitis and synovial pannus formation (Ishikawa et al., 1996). Also, there was a significant sprouting of sympathetic fibers into the upper dermis of
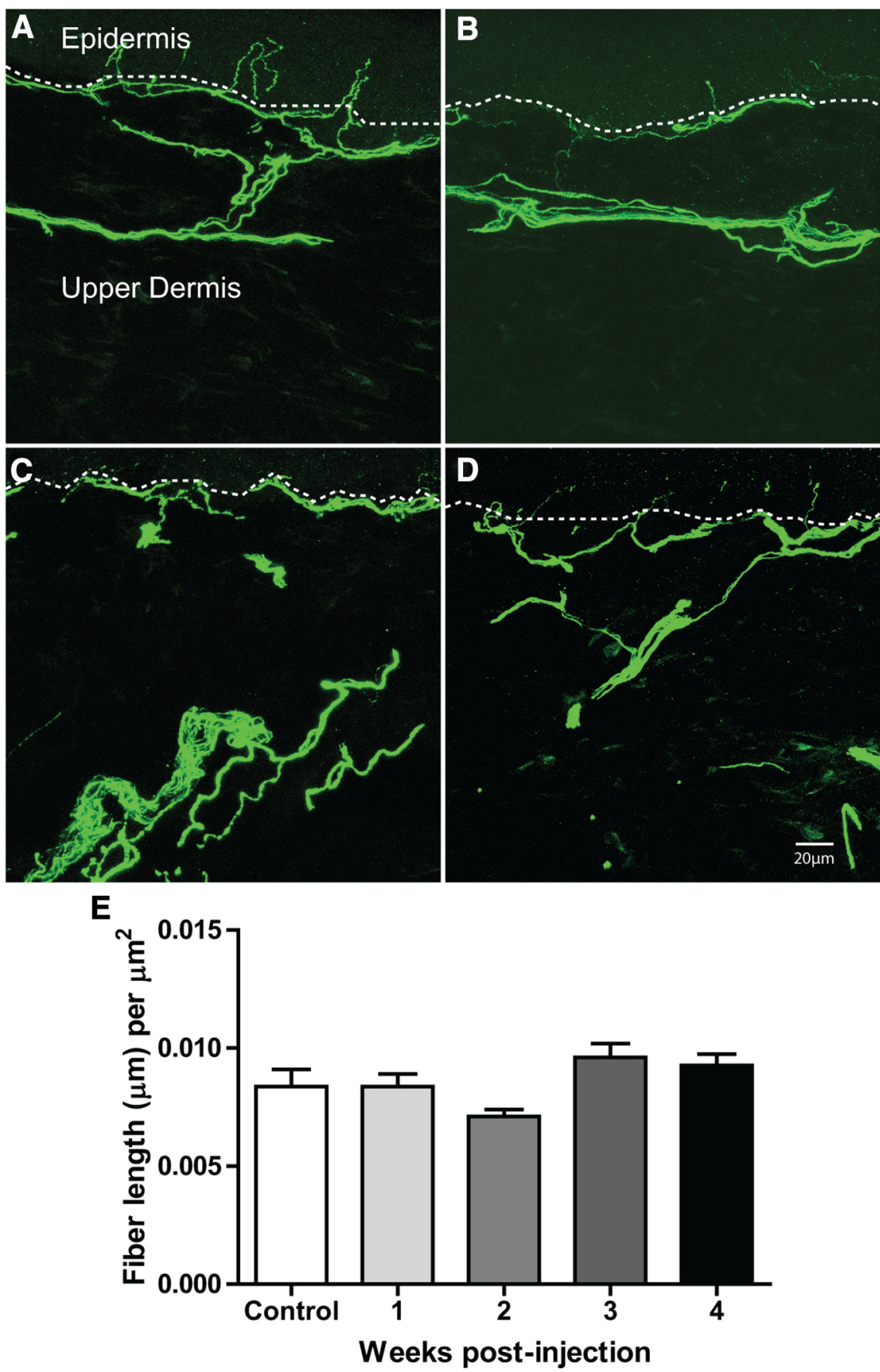

Figure 3. Changes in peptidergic (CGRP-IR) fiber innervation of the glabrous skin adjacent to the joint in CFA-treated rats. $A-D$, Photomicrographs show representative examples of (GRP-IR fiber innervation (green) in the hindpaw skin of the rat in sham animals $(\boldsymbol{A})$, as well as at $2(\boldsymbol{B}), 3(\boldsymbol{C})$, and 4 weeks (D) post-CFA injection. Scale bar, $20 \mu \mathrm{m}$. $\boldsymbol{E}$, Bar graph showing average density of CGRP-IR fibers and fiber bundles in the upper dermis $(n=6, p<0.05)$. No change in (GRP-IR fiber density was observed at any time point. See Materials and Methods for details of quantification. Comparisons were done by means of one-way ANOVA and Bonferroni's post hoc correction. Error bars represent the SEM.

the skin surrounding the inflamed ankle joint. This sprouting was similar to the one we previously observed in the polyarthritis model (Almarestani et al., 2008), supporting the concept of a similar pathology underlying different models of arthritis.

NGF is likely involved both in sympathetic sprouting and in the pain-related behavior. Sympathetic neurons depend on NGF for survival (Levi-Montalcini, 1987) and express its high-affinity receptor trkA (McMahon et al., 2006). NGF levels detected by bioassay are increased in the synovial fluid of patients with rheu- 

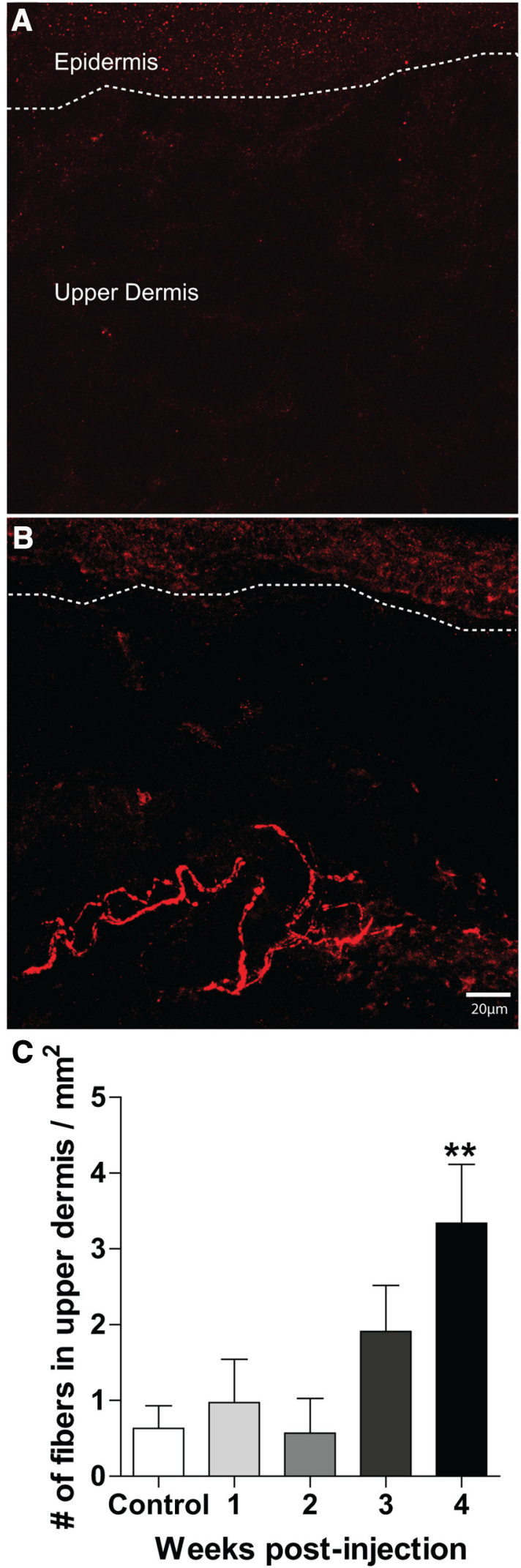

Figure 4. Changes in sympathetic (VMAT-2-IR) fiber innervation in the upper dermis of the skin adjacent to the joint after (FA injection. $A, B$, Representative photomicrographs showing changes in VMAT-2-IR fiber innervation (red) in sham animals $(\boldsymbol{A})$ and CFA-injected animals ( $\boldsymbol{B})$ at the 4 week time point. Scale bar, $20 \mu \mathrm{m}$. C, Bar graph showing average number of sympathetic fibers in the upper dermis at several time points after CFA injection. A significantly higher number of VMAT-2-IR fibers in the upper dermis per scanned area (in square millimeters) was

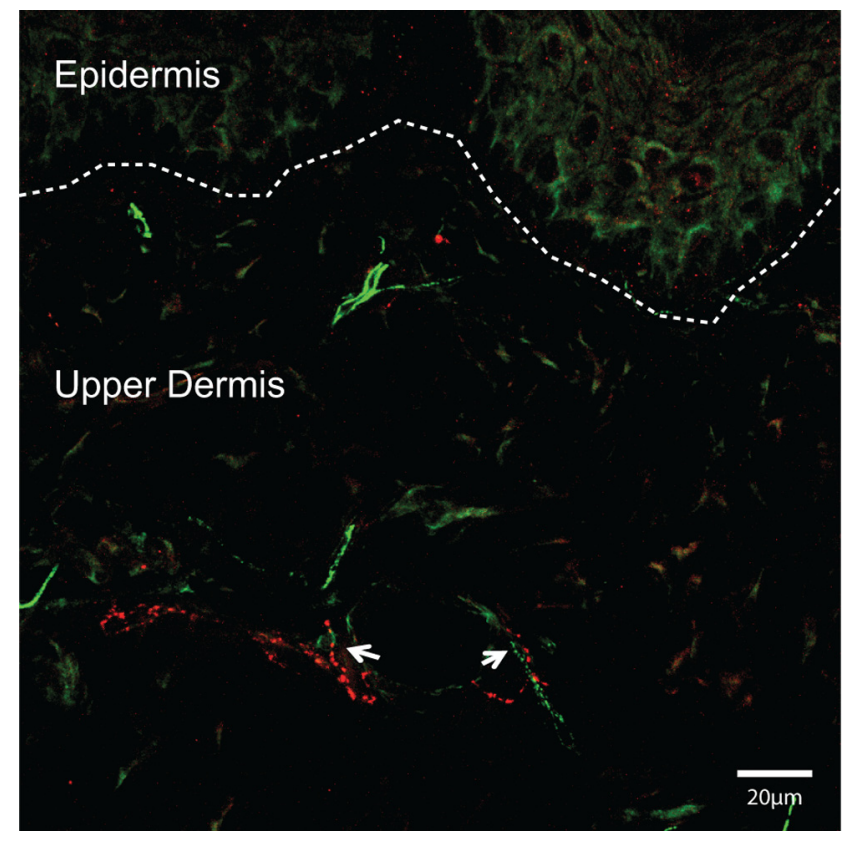

Figure 5. Confocal micrograph illustrating sympathetic and peptidergic fibers in close apposition in the upper dermis of CFA-treated animals. Note that the sympathetic fibers (VMAT2-IR; in green) often run along the same trajectory as the peptidergic fibers (CGRP-IR; in red), suggesting an interaction between the sensory and autonomic fiber populations in the upper dermis.

matoid arthritis compared with normal healthy controls who have an undetectable level of NGF (Aloe et al., 1992; Aloe and Tuveri, 1997). Importantly, in the current study, we detected that the levels of mNGF, but not proNGF, were significantly elevated in the skin at the time point in which we observed the alterations in innervation. We have still unpublished data in this arthritis model showing a significant increase in levels of plasmin, an enzyme that converts proNGF into mNGF, suggesting that the conversion turnover is increased, resulting in an absence of proNGF accumulation and increased levels of mNGF. Therefore, we suggest that chronic inflammation in the joint causes joint and bone destruction accompanied by elevated mNGF levels in surrounding tissues, triggering sympathetic fiber sprouting.

In contrast with the synovium, we found no change in CGRP-IR fiber density in skin of arthritic animals. This result differs from the polyarthritis model, where we observed significant CGRP-IR fiber sprouting beginning at 2 weeks postinjection, but this latter model has obvious skin inflammation besides the joint pathology (Almarestani et al., 2008), likely triggering higher NGF levels in the skin. This reasoning is in agreement with the concept that peptidergic primary afferents are less responsive to NGF changes. In fact, it is well known that sympathetic neurons remain dependent on NGF for survival as well as for maintenance, whereas the postnatal peptidergic neurons cease to require NGF for survival (Gorin and Johnson, 1980; Lewin and Barde, 1996). Skin or mucosa inflammation in other pathological inflammatory diseases, including psoriasis vulgaris, vulvodynia, or interstitial cystitis, induces sensory and/or sympathetic sprouting (Hohenfellner et al., 1992; Nakamura et al., 2003;

detected in the upper dermis in animals at the 4 week post-CFA time point compared with sham animals $\left(n=6,{ }^{* *} p<0.005\right)$. See Materials and Methods for details of quantification. Error bars represent the SEM. 
A
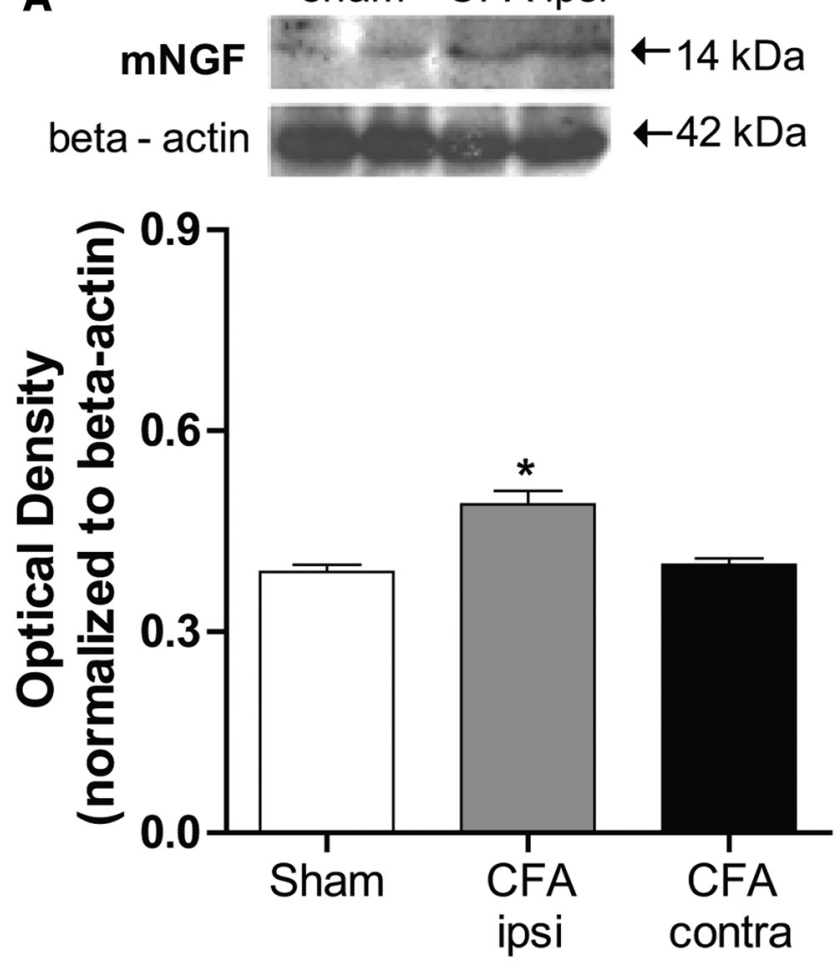

B
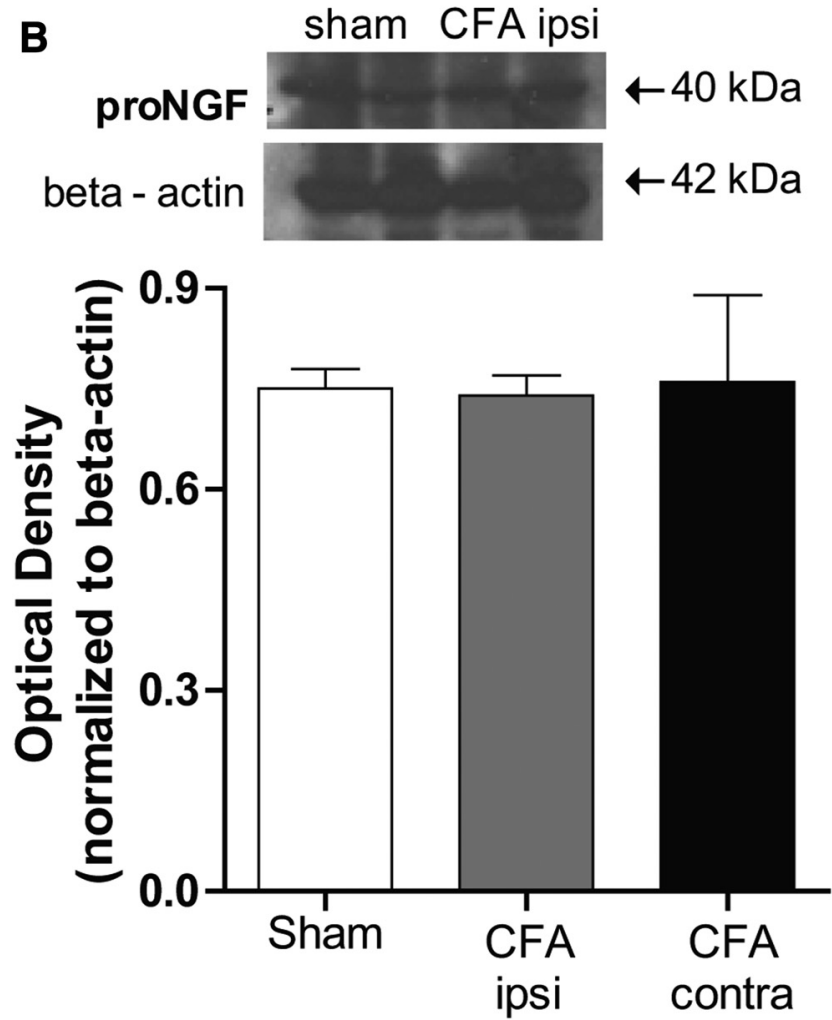

Figure 6. Changes in the protein levels of proNGF and $m$ NGF in the skin of CFA-treated rats. $\boldsymbol{A}, \boldsymbol{B}$, The Western blot analysis performed on the glabrous skin samples 4 weeks after CFA administration showed a significant increase in the protein levels of mNGF $(A)$, with no influence on the protein levels of the precursor proNGF $(\boldsymbol{B})$. Examples of representative Western blots are shown in the top panels in $\boldsymbol{A}$ and $\boldsymbol{B}$. The densitometry results are presented as the mean \pm SEM for all samples. Intergroup differences were analyzed by a one-way ANOVA with a Bonferroni's multiple-comparison test. ${ }^{* *} p<0.001$ indicates a significant difference compared with glabrous skin sample of sham rats.

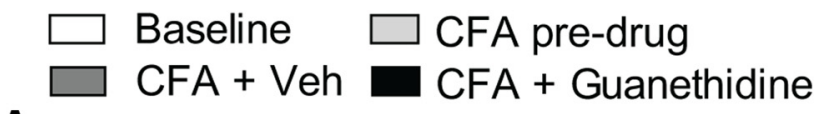

A
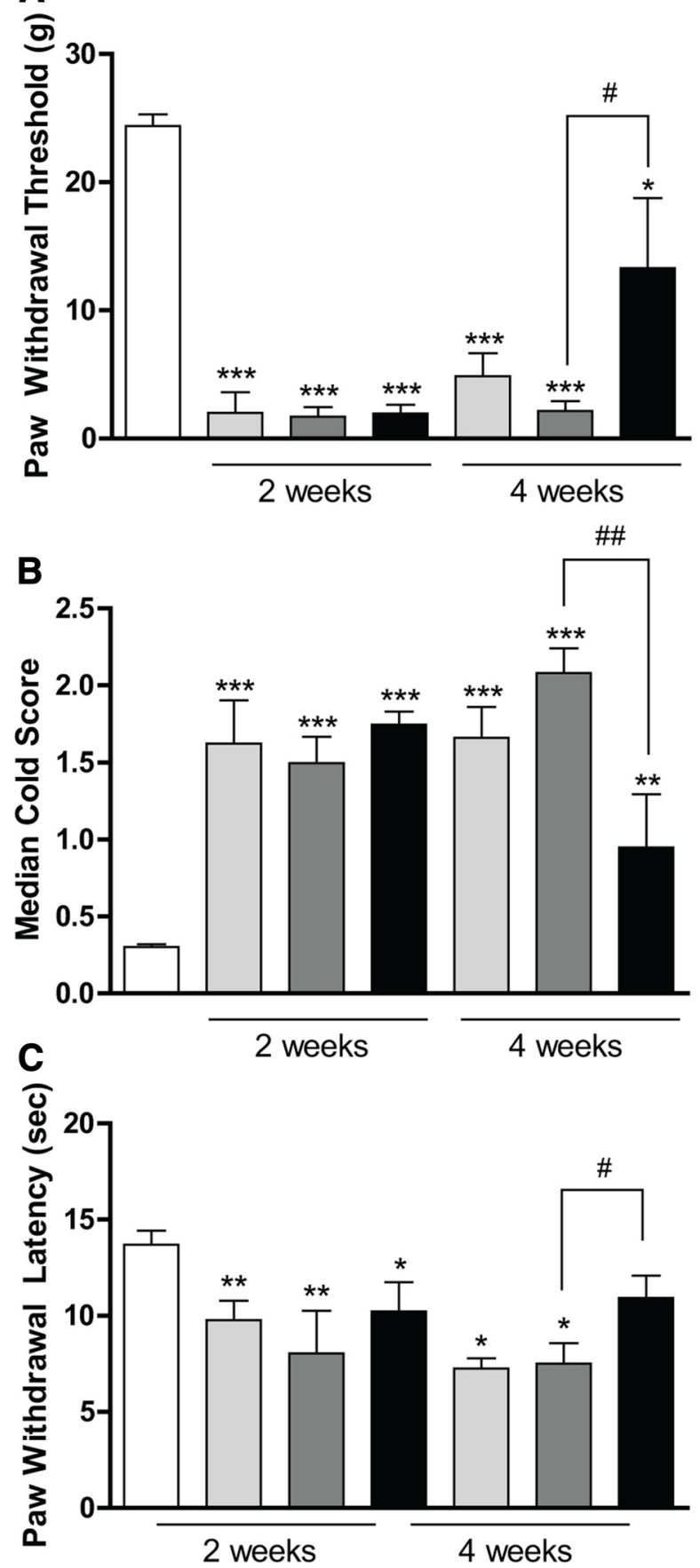

Figure 7. Effect of sympathetic fiber activity suppression with guanethidine on mechanical allodynia, cold allodynia, and heat hyperalgesia in animals at the 2 and 4 week time points post-CFA injection. $A-C$, At the 2 week time point, no statistical difference was found comparing the CFA + vehicle and CFA + guanethidine groups in all three behavioral tests. However, at the 4 week time point, animals from the CFA + guanethidine group displayed a significant attenuation ( $\sim 50 \%$ return to baseline) of mechanical and cold allodynia, respectively, compared with the (FA + vehicle group, which displayed no change (as seen in $\boldsymbol{A}$ and $\boldsymbol{B}$ ). In $\boldsymbol{C}$, it can be observed that guanethidine completely reversed the heat hyperalgesia in (FA-treated rats, with thresholds returning to baseline levels $\left(n=8\right.$ for each group, ${ }^{*} p<0.05,{ }^{* * *} p<0.001$ ). Intergroup differences were analyzed by one-way ANOVA (*significance) with a Bonferroni's multiple-comparison test ( ${ }^{\#}$ CFA vehicle vs guanethidine group statistical difference). Error bars represent the SEM. 
Farmer et al., 2011) and involves different inflammatory components such as infiltration and degranulation of mast cells (Toyoda et al., 1994), neurogenic inflammation (Nakamura et al., 2003), plasma extravasation (Otten et al., 1984), and increased levels of proinflammatory cytokines (Darsow et al., 1997; Nakamura et al., 2003). The above changes are known to induce a substantial increase in NGF expression. Therefore, the moderate changes in mNGF in skin observed in this study may only be sufficient to cause sprouting of sympathetic and not sensory fibers in the skin.

Sympathetic fiber sprouting in the skin in arthritis is comparable to the sprouting previously observed by us in the skin in neuropathic pain models (Grelik et al., 2005; Yen et al., 2006). This presence of ectopic sympathetic fibers in the upper dermis in both arthritis and neuropathic pain models led us to suggest a neuropathic pain component in arthritis (Almarestani et al., 2008), in agreement with the concept that arthritis and neuropathic pain are not two completely separate entities (Bennett, 2006). It is well known that sympathetic efferent activity can affect the firing rate of injured sensory fibers and evoke discharge in silent sensory afferents. In a neuropathic pain model, electrophysiological recordings from DRG neurons revealed a functional coupling of sympathetic and sensory fibers (Chen et al., 1996). In addition, it has been shown that the spontaneous discharge rate of $\mathrm{C}$-fibers is greater when norepinephrine is applied (Xie et al., 1995). In some patients with peripheral neuropathies, sympathetic block results in pain reduction, indicating a role of the sympathetic fibers (Roberts, 1986). We observed that in inflammatory arthritis, as in neuropathic pain models, sympathetic fibers in the upper dermis wrapped around peptidergic fibers, instead of innervating blood vessels (Almarestani et al., 2008; current study). Also, in the synovial membrane, sensory/sympathetic fiber appositions were more abundant in the CFA group. These abnormal fiber arrangements would favor an effect of transmitters/modulators released from the sympathetic fibers on primary afferent excitability. To confirm this, we blocked the function of sympathetic fibers using guanethidine. We detected that at the 4 week time point, guanethidine-treated arthritic rats had an amelioration of mechanical and cold allodynia thresholds, and a complete suppression of heat hypersensitivity. Conversely, in all three behavioral tests, no differences in thresholds were found between sham or CFA animals injected with vehicle or guanethidine at the 2 week time point. These results are not surprising since ectopic sympathetic fibers only become significantly present at 4 weeks after CFA injection. Based on our data and that of others (Ghilardi et al., 2012), we suggest that guanethidine suppresses the abnormal sympathetic activity in this arthritis model in both skin and joint. Our data following guanethidine administration is comparable to that obtained in other chronic pain models (Malmberg and Basbaum, 1998; Xanthos et al., 2008). Suppressing the sympathetic nervous system increases skin blood flow and temperature (Yanagiya et al., 1999) and alters bone architecture in adult growing rats (Pagani et al., 2008). Furthermore, clinical observations indicate that the sympathetic nervous system plays a role in the pathogenesis of inflammation (Kozin et al., 1976; Levine et al., 1986). These changes could explain in part the amelioration of pain-related behavior that we observed. However, no guanethidine-induced behavioral changes were observed at the 2 week time point or in non-CFA-treated rats, suggesting they are the result of the suppression of the pronociceptive effect of the ectopic sympathetic fibers.

However, guanethidine treatment induced a partial amelioration of the pain-related behavior at 4 weeks, indicating that other factors may contribute to the pain, such as persistent inflamma- tion and bone and cartilage damage. Clinically, the persistence of synovitis and pain in patients with osteoarthritis is explained by the increased innervation by peptidergic free nerve endings releasing high amounts of substance P (Saito and Koshino, 2000). This increased peptidergic innervation in joints has also been found in animal models (Imai et al., 1997; Ghilardi et al., 2012). The increased activation of peptidergic primary afferents leads to neurogenic inflammation, which plays an important role in arthritis (Levine et al., 1985). Importantly, through neurogenic inflammation, the skin surrounding the joints will also get inflamed (Ahmed et al., 1995). This phenomenon leads to activation of immune cells and mast cells in the surrounding tissues, amplifying the inflammatory cascade and resulting in increased release of NGF. This peripherally produced NGF is sufficient to maintain the sensitization of nociceptive sensory neurons and induce inflammatory hyperalgesia (McMahon et al., 1995; Rueff et al., 1996), besides inducing sympathetic sprouting, which plays a role in pain in itself as documented here.

In conclusion, we show in an animal model of monoarthritis a persistent pain hypersensitivity accompanied by an abnormal sprouting of sympathetic fibers in the skin adjacent to the inflamed joint. This abnormal growth of sympathetic fibers was accompanied by elevated levels of mNGF, but not of its precursor proNGF, making the modulation of mNGF levels an interesting target for the treatment of inflammatory arthritis. We also observed that the suppression of sympathetic fiber function led to an amelioration of the pain-related behavior. These data reinforce the concept of a neuropathic component in arthritis and that sympathetic fibers play a role in the genesis of the pain associated with arthritis.

\section{References}

Ahmed M, Bjurholm A, Schultzberg M, Theodorsson E, Kreicbergs A (1995) Increased levels of substance $\mathrm{P}$ and calcitonin gene-related peptide in rat adjuvant arthritis. A combined immunohistochemical and radioimmunoassay analysis. Arthritis Rheum 38:699-709. CrossRef Medline

Allard S, Leon WC, Pakavathkumar P, Bruno MA, Ribeiro-da-Silva A, Cuello AC (2012) Impact of the NGF maturation and degradation pathway on the cortical cholinergic system phenotype. J Neurosci 32:2002-2012. CrossRef Medline

Almarestani L, Longo G, Ribeiro-da-Silva A (2008) Autonomic fiber sprouting in the skin in chronic inflammation. Mol Pain 4:56. CrossRef Medline

Almarestani L, Fitzcharles MA, Bennett GJ, Ribeiro-da-Silva A (2011) Imaging studies in Freund's complete adjuvant model of regional polyarthritis, a model suitable for the study of pain mechanisms, in the rat. Arthritis Rheum 63:1573-1581. CrossRef Medline

Aloe L, Tuveri MA (1997) Nerve growth factor and autoimmune rheumatic diseases. Clin Exp Rheumatol 15:433-438. Medline

Aloe L, Tuveri MA, Carcassi U, Levi-Montalcini R (1992) Nerve growth factor in the synovial fluid of patients with chronic arthritis. Arthritis Rheum 35:351-355. CrossRef Medline

Bennett GJ (2006) Can we distinguish between inflammatory and neuropathic pain? Pain Res Manag 11:11A-15A.

Breivik H, Collett B, Ventafridda V, Cohen R, Gallacher D (2006) Survey of chronic pain in Europe: prevalence, impact on daily life, and treatment. Eur J Pain 10:287-333. CrossRef Medline

Bruno MA, Cuello AC (2006) Activity-dependent release of precursor nerve growth factor, conversion to mature nerve growth factor, and its degradation by a protease cascade. Proc Natl Acad Sci U S A 103:6735-6740. CrossRef Medline

Butler SH, Godefroy F, Besson JM, Weil-Fugazza J (1992) A limited arthritic model for chronic pain studies in the rat. Pain 48:73-81. CrossRef Medline

Chen Y, Michaelis M, Janig W, Devor M (1996) Adrenoreceptor subtype mediating sympathetic-sensory coupling in injured sensory neurons. J Neurophysiol 76:3721-3730. Medline

Choi Y, Yoon YW, Na HS, Kim SH, Chung JM (1994) Behavioral signs of 
ongoing pain and cold allodynia in a rat model of neuropathic pain. Pain 59:369-376. CrossRef Medline

Darsow U, Scharein E, Bromm B, Ring J (1997) Skin testing of the pruritogenic activity of histamine and cytokines (interleukin-2 and tumour necrosis factor-alpha) at the dermal-epidermal junction. Br J Dermatol 137: 415-417. Medline

Farmer MA, Taylor AM, Bailey AL, Tuttle AH, MacIntyre LC, Milagrosa ZE, Crissman HP, Bennett GJ, Ribeiro-da-Silva A, Binik YM, Mogil JS (2011) Repeated vulvovaginal fungal infections cause persistent pain in a mouse model of vulvodynia. Sci Transl Med 3:101-191.

Flatters SJ, Bennett GJ (2004) Ethosuximide reverses paclitaxel- and vincristine-induced painful peripheral neuropathy. Pain 109:150-161. CrossRef Medline

Ghilardi JR, Freeman KT, Jimenez-Andrade JM, Coughlin KA, Kaczmarska MJ, Castaneda-Corral G, Bloom AP, Kuskowski MA, Mantyh PW (2012) Neuroplasticity of sensory and sympathetic nerve fibers in a mouse model of a painful arthritic joint. Arthritis Rheum 64:2223-2232. CrossRef Medline

Gorin PD, Johnson EM Jr (1980) Effects of long-term nerve growth factor deprivation on the nervous system of the adult rat: an experimental autoimmune approach. Brain Res 198:27-42. CrossRef Medline

Grelik C, Bennett GJ, Ribeiro-da-Silva A (2005) Autonomic fibre sprouting and changes in nociceptive sensory innervation in the rat lower lip skin following chronic constriction injury. Eur J Neurosci 21:2475-2487. CrossRef Medline

Hara-Irie F, Amizuka N, Ozawa H (1996) Immunohistochemical and ultrastructural localization of CGRP-positive nerve fibers at the epiphyseal trabecules facing the growth plate of rat femurs. Bone 18:29-39. CrossRef Medline

Henry JL (2004) Molecular events of chronic pain: from neuron to whole animal in an animal model of osteoarthritis. Novartis Found Symp 260: 139-145. CrossRef Medline

Hohenfellner M, Nunes L, Schmidt RA, Lampel A, Thüroff JW, Tanagho EA (1992) Interstitial cystitis: increased sympathetic innervation and related neuropeptide synthesis. J Urol 147:587-591. Medline

Imai S, Tokunaga Y, Konttinen YT, Maeda T, Hukuda S, Santavirta S (1997) Ultrastructure of the synovial sensory peptidergic fibers is distinctively altered in different phases of adjuvant induced arthritis in rats: ultramorphological characterization combined with morphometric and immunohistochemical study for substance $\mathrm{P}$, calcitonin gene related peptide, and protein gene product 9.5. J Rheumatol 24:2177-2187. Medline

Ishikawa H, Hirata S, Andoh Y, Kubo H, Nakagawa N, Nishibayashi Y, Mizuno K (1996) An immunohistochemical and immunoelectron microscopic study of adhesion molecules in synovial pannus formation in rheumatoid arthritis. Rheumatol Int 16:53-60. CrossRef Medline

Kim SH, Na HS, Sheen K, Chung JM (1993) Effects of sympathectomy on a rat model of peripheral neuropathy. Pain 55:85-92. CrossRef Medline

Kozin F, McCarty DJ, Sims J, Genant H (1976) The reflex sympathetic dystrophy syndrome. I. Clinical and histologic studies: evidence for bilaterality, response to corticosteroids and articular involvement. Am J Med 60:321-331. CrossRef Medline

Lawrence RC, Felson DT, Helmick CG, Arnold LM, Choi H, Deyo RA, Gabriel S, Hirsch R, Hochberg MC, Hunder GG, Jordan JM, Katz JN, Kremers HM, Wolfe F (2008) Estimates of the prevalence of arthritis and other rheumatic conditions in the United States. Part II. Arthritis Rheum 58:26-35. CrossRef Medline

Levi-Montalcini R (1987) The nerve growth factor 35 years later. Science 237:1154-1162. CrossRef Medline

Levine JD, Moskowitz MA, Basbaum AI (1985) The contribution of neurogenic inflammation in experimental arthritis. J Immunol 135:843s-847s. Medline

Levine JD, Fye K, Heller P, Basbaum AI, Whiting-O’Keefe Q (1986) Clinical response to regional intravenous guanethidine in patients with rheumatoid arthritis. J Rheumatol 13:1040-1043. Medline

Lewin GR, Barde YA (1996) Physiology of the neurotrophins. Annu Rev Neurosci 19:289-317. CrossRef Medline

Mach DB, Rogers SD, Sabino MC, Luger NM, Schwei MJ, Pomonis JD, Keyser CP, Clohisy DR, Adams DJ, O'Leary P, Mantyh PW (2002) Origins of skeletal pain: sensory and sympathetic innervation of the mouse femur. Neuroscience 113:155-166. CrossRef Medline

Malmberg AB, Basbaum AI (1998) Partial sciatic nerve injury in the mouse as a model of neuropathic pain: behavioral and neuroanatomical correlates. Pain 76:215-222. CrossRef Medline

Maxwell RA, Plummer AJ, Schneider F, Povalski H, Daniel AI (1960) Pharmacology of [2-(octahydro-1-azocinyl)-ethyl]-guanidine sulfate ( $\mathrm{Su}$ 5864). J Pharmacol Exp Ther 128:22-29. Medline

McMahon SB, Bennett DL, Priestley JV, Shelton DL (1995) The biological effects of endogenous nerve growth factor on adult sensory neurons revealed by a trkA-IgG fusion molecule. Nat Med 1:774-780. CrossRef Medline

McMahon SB, Bennett DL, Bevan S, eds (2006) Inflammatory mediators and modulators of pain. London: Elsevier Churchill Livingstone.

Miller RJ, Jung H, Bhangoo SK, White FA (2009) Cytokine and chemokine regulation of sensory neuron function. Handb Exp Pharmacol 417-449. CrossRef Medline

Nagakura Y, Okada M, Kohara A, Kiso T, Toya T, Iwai A, Wanibuchi F, Yamaguchi T (2003) Allodynia and hyperalgesia in adjuvant-induced arthritic rats: time course of progression and efficacy of analgesics. J Pharmacol Exp Ther 306:490-497. CrossRef Medline

Nakamura M, Toyoda M, Morohashi M (2003) Pruritogenic mediators in psoriasis vulgaris: comparative evaluation of itch-associated cutaneous factors. Br J Dermatol 149:718-730. CrossRef Medline

Osikowicz M, Mika J, Makuch W, Przewlocka B (2008) Glutamate receptor ligands attenuate allodynia and hyperalgesia and potentiate morphine effects in a mouse model of neuropathic pain. Pain 139:117-126. CrossRef Medline

Otten U, Baumann JB, Girard J (1984) Nerve growth factor induces plasma extravasation in rat skin. Eur J Pharmacol 106:199-201. CrossRef Medline

Pagani F, Sibilia V, Cavani F, Ferretti M, Bertoni L, Palumbo C, Lattuada N, De Luca E, Rubinacci A, Guidobono F (2008) Sympathectomy alters bone architecture in adult growing rats. J Cell Biochem 104:2155-2164. CrossRef Medline

Roberts WJ (1986) A hypothesis on the physiological basis for causalgia and related pains. Pain 24:297-311. CrossRef Medline

Rueff A, Dawson AJ, Mendell LM (1996) Characteristics of nerve growth factor induced hyperalgesia in adult rats: dependence on enhanced bradykinin-1 receptor activity but not neurokinin-1 receptor activation. Pain 66:359-372. CrossRef Medline

Saito T, Koshino T (2000) Distribution of neuropeptides in synovium of the knee with osteoarthritis. Clin Orthop Relat Res 376:172-182. Medline

Taylor AM, Peleshok JC, Ribeiro-da-Silva A (2009) Distribution of P2X(3)immunoreactive fibers in hairy and glabrous skin of the rat. J Comp Neurol 514:555-566. CrossRef Medline

Toyoda M, Zhang X, Petrosian A, Galera OA, Wang SJ, Jordan SC (1994) Modulation of immunoglobulin production and cytokine mRNA expression in peripheral blood mononuclear cells by intravenous immunoglobulin. J Clin Immunol 14:178-189. CrossRef Medline

Vermeirsch H, Biermans R, Salmon PL, Meert TF (2007) Evaluation of pain behavior and bone destruction in two arthritic models in guinea pig and rat. Pharmacol Biochem Behav 87:349-359. CrossRef Medline

Xanthos DN, Bennett GJ, Coderre TJ (2008) Norepinephrine-induced nociception and vasoconstrictor hypersensitivity in rats with chronic postischemia pain. Pain 137:640-651. CrossRef Medline

Xie Y, Zhang J, Petersen M, LaMotte RH (1995) Functional changes in dorsal root ganglion cells after chronic nerve constriction in the rat. J Neurophysiol 73:1811-1820. Medline

Yanagiya Y, Yoshimura R, Hori M, Kuwahara M, Tsubone H, Sugano S (1999) The influence of chronic sympathectomy on cutaneous blood flow in the rat tail. J Vet Med Sci 61:795-801. CrossRef Medline

Yen LD, Bennett GJ, Ribeiro-da-Silva A (2006) Sympathetic sprouting and changes in nociceptive sensory innervation in the glabrous skin of the rat hind paw following partial peripheral nerve injury. J Comp Neurol 495: 679-690. CrossRef Medline

Zimmermann M (1983) Ethical guidelines for investigations of experimental pain in conscious animals. Pain 16:109-110. CrossRef Medline 